\title{
Colangiopatía asociada al VIH: un desafío diagnóstico, presentación de caso clínico y revisión de la literatura
}

HIV-associated cholangiopathy: a diagnostic challenge, clinical case presentation and literature review

Barzallo Sánchez David Esteban, Martínez Gaona Karla del Cisne ${ }^{2}$

VOLUMEN 38 | N 3 | DICIEMBRE 2020

FECHA DE RECEPCIÓN: 19/05/2020 FECHA APROBACIÓN: 01/04/2021 FECHA DE PUBLICACIÓN: 06/04/2021

1. Hospital de Especialidades José Carrasco Arteaga. Unidad de Cirugía General. Cuenca - Ecuador

1. Universidad de Cuenca. Egresada del Posgrado de

Cirugía General.

Cuenca - Ecuador

\section{Caso \\ Clínico \\ Clinical Case}

DOI: https://doi.org/10.18537/RFCM.38.03.08

Correspondencia: davidbarza@gmail.com

Dirección:

Nicanor Aguilar y Avenida Solano

Código Postal:

010150

Teléfono:

2861500

Cuenca - Ecuador

\section{RESUMEN}

Introducción: la infección por el virus de inmunodeficiencia humana $(\mathrm{VIH})$ produce una estasis biliar secundaria a un daño estructural de las vías biliares.

Caso clínico: paciente masculino de 53 años con antecedentes de VIH y diabetes mellitus tipo 2, acude con dolor cólico en hipocondrio izquierdo, deposiciones diarreicas, pérdida de peso, coluria, ictericia, patrón colestásico elevado y uroanálisis infeccioso. En la Colangiopancreatografía retrógrada endoscópica (CPRE) se evidencia un lito en colédoco más estenosis intrínseca, se realiza una esfinterectomía y colocación de stent. Es valorado por el servicio de cirugía hepato pancreatobiliar y se diagnostica de colangiopatía asociada al VIH. Después del CPRE se da un adecuado control del dolor abdominal y disminución del patrón colestásico. Se decide alta en buenas condiciones y seguimiento ambulatorio.

Conclusión: en un paciente con VIH más ictericia obstructiva se debe sospechar en colangiopatías asociadas; la CPRE es el método de elección para el diagnóstico y tratamiento.

Palabras clave: colangiopancreatografía retrógrada endoscópica, colestasis, conductocoledoco, síndrome de inmunodeficiencia adquirida. 


\section{ABSTRACT}

Introduction: infection by the human immunodeficiency virus (HIV) produces biliary stasis secondary to structural damage to the bile ducts.

Clinical Case: a 53-year-old male with a history of HIV and type 2 diabetes mellitus, presented with colicky pain in the left upper quadrant, diarrheal stools, weight loss, coluria, jaundice, an elevated cholestatic pattern, and infectious urinalysis. Endoscopic retrograde cholangiopancreatography (ERCP) revealed a common bile duct stone plus intrinsic stenosis, a sphincterectomy and stent placement were performed. The pacient was evaluated by the hepato pancreatobiliary surgery service and diagnosed with HIV-associated cholangiopathy. After ERCP, there is adequate control of abdominal pain and a decrease in the cholestatic pattern. Discharge is decided in good condition and outpatient follow-up.

Conclusion: a patient with HIV plus obstructive jaundice, it should be suspected in associated cholangiopathies; ERCP is the method of choice for diagnosis and treatment.

Key words: cholangipancreatography endoscopic retrograde, cholestasis, common bill duct, acquired immunodeficiency syndromem.

\section{INTRODUCCIÓN}

El VIH cambia en gran medida la fisiología del organismo humano, primero por vulnerar los mecanismos de defensa y segundo por los efectos adversos causados tras el tratamiento con antirretrovirales a largo plazo; dentro de la alteración funcional de los órganos, se encuentra alteración de la función hepática y de manera conjunta estasis biliar secundaria al daño estructural de las vías biliares presentando una mortalidad del $14 \%$ al $18 \%$ aproximadamente. Estos cuadros clínicos se definen con colangiopatías asociadas a $\mathrm{VIH}$ y deben ser identificados en contraste a procesos neoplásicos de origen biliar o de órganos vecinos ${ }^{1-3}$.

No existen estadísticas descritas sobre la prevalencia de estas colangiopatías, más ante un cuadro de colangiopatías asociadas a VIH se ha descrito la presencia de gérmenes oportunistas que pueden causar colangitis y dar una morbilidad elevada a estos pacientes ${ }^{3}$.

El tratamiento de esta patología va encaminado a disminuir los síntomas secundarios a la estasis biliar. Si pese al tratamiento instaurado el paciente no tiene mejoría clínica, puede estar en relación a una resistencia a la terapia con antirretrovirales ${ }^{1,2,4}$. El objetivo del presente trabajo es presentar un caso clínico con diagnóstico de VIH y sus alteraciones biliares acompañado de la revisión de la literatura existente.

\section{PRESENTACIÓN DEL CASO}

Se trata de un paciente de sexo masculino de 53 años con antecedentes de VIH desde hace 26 años, diabetes mellitus hace 1 año, refiere presentar desde hace 45 días dolor abdominal tipo cólico de moderada intensidad localizado en hipocondrio izquierdo acompañado de deposiciones blandas y pérdida no intencional de peso no cuantificado. Hace 1 mes se suma coluria e ictericia en escleras por lo que es valorado en casa de salud particular en donde recibe el diagnóstico de pancreatitis aguda con coledocolitiasis. Su infección por VIH estaba tratada con tenofovir, emtricitabina y efavirenz, en su último control de carga viral fue indetectable. Para su diabetes recibe metformina. Durante la valoración física sus constantes vitales se encontraban dentro de rangos normales, regular aspecto general, 
ictericia generalizada, no adenopatías, a nivel de abdomen dolor a la palpación profunda en mesogastrio y no presenta signos de irritación peritoneal.

Los exámenes complementarios revelaron una hiperbilirrubinemia de 19.9 mg/dl con predominio de la fracción directa $(16.11 \mathrm{mg} / \mathrm{dl})$, fosfatasa alcalina $1384 \mathrm{U} / \mathrm{L}$ y gamaglutamiltransferasa en $4160 \mathrm{U} / \mathrm{L}$, amilasa $407 \mathrm{U} / \mathrm{L}$. Elemental y microscópico de orina con nitritos positivo. Recuento de linfocitos CD3: 1681.79 células $/ \mathrm{mm}^{3}, \quad$ CD4: 728.79 células $/ \mathrm{mm}^{3}$, CD8: 823.15 células $/ \mathrm{mm}^{3}$.

CPRE: colédoco dilatado (22 mm), en su extremo distal se observa imagen de vacío de señal de 7.4 × 5 mm sugerente de lito; además, engrosamiento e irregularidad de la pared del conducto colédoco con disminución de la luz a considerar proceso ocupativo (ver Imagen $\mathrm{N}^{\circ} 1$ ).

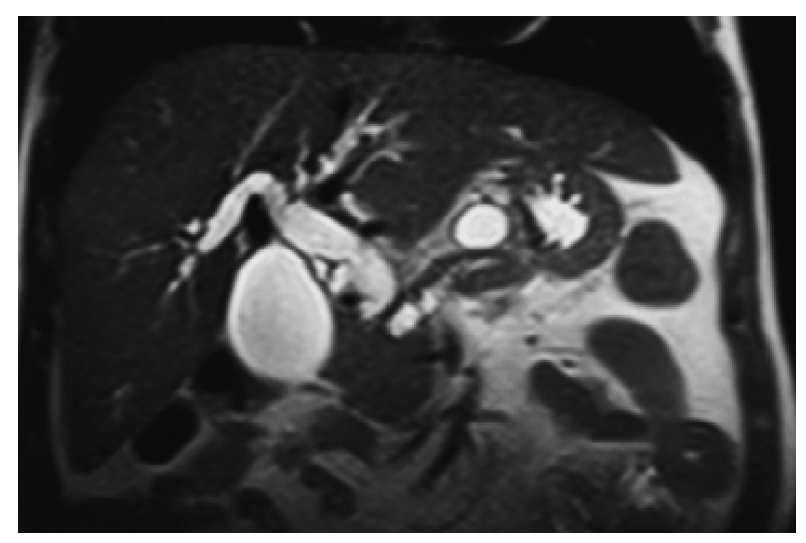

Imagen $\mathrm{N}^{\circ} 1$

CPRE. Extremo distal de colédoco con vacío de señal en relación a lito y disminución de la luz. Vías biliares intra y extrahepáticas dilatadas.

Con estos hallazgos es interconsultado el equipo de gastroenterología, servicio que decide realizar una CPRE evidenciando estenosis a nivel de tercio inferior del colédoco con longitud de $35 \mathrm{~mm}$ de tipo intrínseco aparentemente, por lo que se realizó una esfinterectomía y colocación de prótesis metálica auto expandible cubierta de $40 \mathrm{~mm}$ de longitud, por $10 \mathrm{~mm}$ de diámetro (ver Imagen $N^{\circ}$ 2). No fue posible la toma de biopsia.

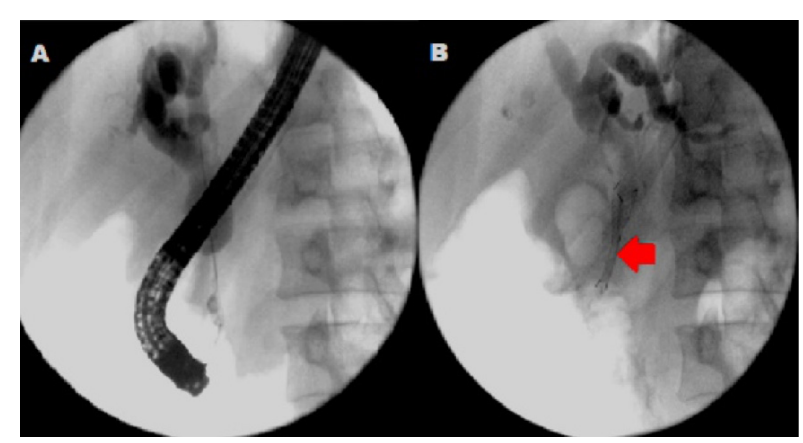

Imagen $\mathrm{N}^{\circ} 2$

CPRE. A. estenosis del colédoco impresiona por posible patología intrínseca. B. colangiografía tras la colocación de stent (prótesis mecánica).

Posterior a este manejo y con la sospecha de masa tumoral que ocasione la estenosis se realiza tomografía trifásica de abdomen más tomografía simple y contrastada de tórax: hígado homogéneo, neumobilia, ausencia de dilatación de la vía biliar intrahepática, colédoco con la presencia de stent en su luz. Páncreas de aspecto normal sin lesiones ni captación anómala de contraste, con dilatación del conducto principal $5.7 \mathrm{~mm}$, adenopatías para - aórticas e intercavo - aorticas de 5 y 8 mm, tórax sin lesiones.

Durante su evolución el paciente presentó un descenso de bilirrubinas de una bilirrubina total de $19.1 \mathrm{mg} / \mathrm{dl}$, bilirrubina directa de $16.11 \mathrm{mg} / \mathrm{dl}$ e indirecta de $3.08 \mathrm{mg} / \mathrm{dl}$ a valores de 6.85, 5.92 y $0.93 \mathrm{mg} / \mathrm{dl}$ respectivamente; de igual manera hay un descenso del marcador tumoral $\mathrm{Ca}$ 19.9 que previo al procedimiento endoscópico se encontraba en valores de $213 \mathrm{U} / \mathrm{ml}$ y posteriormente descienden a $143 \mathrm{U} / \mathrm{ml}$.

Recibió tratamiento antibiótico, se realizó la valoración por el servicio de cirugía hepato pancreato biliar en el contexto de la sospecha 
de masa tumoral que requiera manejo quirúrgico, llevando el caso a comité, tras la revisión de las imágenes y el contexto del caso en particular se sugiere el diagnóstico de colangiopatía asociada al VIH, como causa de la estenosis ante la ausencia de imágenes que identifiquen masa tumoral a nivel de la encrucijada pancreato biliar, dejando al paciente en vigilancia en un contexto ambulatorio.

\section{DISCUSIÓN}

Dentro del grupo de las colangiopatías, el virus de inmunodeficiencia humano y su tratamiento antirretroviral tienen efectos pro inflamatorios a nivel de hígado y vías biliares que consecuentemente lleva a falla hepática asociadas a una mortalidad entre $14 \%$ a $18 \%$ 1,2. Según Harshad y colaboradores ${ }^{1}$ reportan una ventana de tiempo entre el diagnóstico de VIH y la presencia de la colangiopatía asociada al SIDA de 3 años aproximadamente. No se conoce una prevalencia e incidencia real de esta patología, más debe ser tomada en cuenta de manera prioritaria en pacientes con VIH y sintomatología biliar dentro del diagnóstico diferencial ante la sospecha de procesos neoplásicos de la zona1,2. En la clasificación de las enfermedades del hígado por $\mathrm{VIH}$ está el primer grupo en donde se encuentran patologías asociadas a la inmunosupresión (colangiopatía por SIDA, la colecistitis alitiásica, las neoplasias relacionadas con el SIDA).

Los investigadores Naseer y colaboradores ${ }^{3}$ año 2018 y Romero y colaboradores ${ }^{6}$ en el año 2016 señalan en sus publicaciones que la colangiopatía asociada al VIH es causada por una estrechez del tracto biliar y daño hepático colestásico, cuadro que es descrito por primera vez en 1983 por Pitlik, Guarda y colaboradores profundizando su investigación en pacientes con infección por VIH y dolor en hipocondrio derecho encontrando varios patógenos oportunistas que condicionan la posterior colangiopatía. A su vez se ha encontrado cuatro patrones colangiográficos que se asemejan a la colangitis esclerosante primaria: estenosis papilar, colecistitis alitiásica, estenosis extra hepáticas y lesiones esclerosantes intra y extra hepáticas. El Cryptosporidium parvum (C. parvum) se encuentra como oportunista entre un $20 \%$ a $57 \%$ de infecciones del árbol biliar ${ }^{3,5}$. Entre otros patógenos asociados está el Histoplasma capsulatum, Citomegalovirus, Enterocytozoon bieneusi y las Isosporas ${ }^{3,5}$.

La sintomatología deriva de la colestasis por la estrechez del esfínter de Oddi y estenosis de la vía biliar. La colestasis se define como la detención parcial o total al flujo biliar en donde unas de sus causas es la colangitis asociada al $\mathrm{VIH}^{6}$. Las manifestaciones clínicas de la colangiopatía asociada a VIH suelen presentar signos y síntomas en un 20\% de pacientes infectados pero inmunocompetentes con recuentos CD4 altos $^{7}$ dependen de la obstrucción completa del flujo biliar, en donde el $90 \%$ de pacientes presentan dolor agudo a nivel de epigastrio que se irradia a hipocondrio derecho, náuseas, vómitos más una progresiva ictericia, coluria y acolia ${ }^{5}$. Se debe tener en cuenta la presencia de fiebre o compromiso del estado general lo que indica una colangitis o pancreatitis asociada; es poco común el cuadro de pancreatitis asociada a colangiopatía por $\mathrm{VIH} \mathrm{H}^{1,6}$.

En el panel de laboratorio clínico es frecuente la elevación de las enzimas fosfatasa alcalina y gamaglutamiltransferasa asociada a una elevación discreta de transaminasas hepáticas. La elevación de la bilirrubina viene en relación directa con el grado de obstrucción biliar la misma que puede ser parcial, completa o intermitente; frente a obstrucciones de mayor tiempo de evolución el paciente presenta 
alteraciones en sus tiempos de coagulación por el déficit de vitamina $\mathrm{K}$, pero al contrario a la insuficiencia sintética hepatocelular, en cuadros colestásico hay una respuesta favorable al suplemento vitamínico6. La colangiopatía asociada al SIDA ${ }^{8}$ suele manifestarse cuando los niveles de linfocitos T CD4 están menores a 100 células por $\mathrm{mm}^{3}$.

La ecografía abdominal se considera el estudio de imagen de primera elección para una exploración inicial no invasiva de las vías biliares con una sensibilidad del $97 \%$ y especificidad del $100 \%$ en una colangiopatía asociada a $\mathrm{VIH}^{1}$. Consiste en ser un estudio de bajo costo, accesible, rápido que no produce complicaciones en el paciente, permite valorar la causa de la obstrucción o la presencia de signos indirectos de obstrucción como la vía biliar intra y extra hepática dilatada. Se debe tener en consideración que este estudio es operador dependiente por lo que podría dar resultados falsos negativos en caso de fases tempranas de la obstrucción o falsos positivos en dilataciones fisiológicas de la vía biliar en adultos mayores o pacientes postcolecistectomía ${ }^{6}$.

Si la ecografía abdominal no aporta mayores datos colestásicos, se debe realizar una colangiografía por resonancia magnética o una ecoendoscopía, estas pruebas tienen una precisión alta para la identificación de colestasis extra hepática intraluminal ${ }^{3,7,9}$.

La colangioresonancia al ser un estudio no invasivo es considerado el método de primera elección para el diagnóstico de colangitis esclerosante primaria y colangiopatías difusas asociadas a $\mathrm{VIH}$, en donde el signo típico es la presencia de estenosis papilar más una estenosis cónica de la porción distal del colédoco y dilataciones segmentarias o saculares más engrosamiento de la pared ductal3,8. La ecoendoscopía permite realizar la toma de biopsias de masas al servir de guía para su punción. En caso de sospecha de malignidad se recomienda una tomografía computarizada, la misma que puede servir de guía para la toma de biopsias, determinar las intervenciones paliativas y el estudio de metástasis regionales o a distancia.

La CPRE se considera un estudio diagnóstico terapéutico invasivo que permite la toma de biopsias, drenaje de cálculos y colocación de prótesis o drenajes; en colangiopatías asociadas a VIH tiene una sensibilidad de $97 \%$ y especificidad de $100 \% 3,6$, en este procedimiento se recomienda realizar una esfinterectomía de la ampolla de Vater para disminuir el dolor abdominal en los pacientes ${ }^{10}$. Tonolini et $\mathrm{al}^{7}$ describen en su artículo "Colangiopatía relacionada con el VIH/SIDA: revisión pictórica con énfasis en Resultados de MRCP y diagnóstico diferencial" cuatro tipos de variantes de la vía biliar descritas en la Tabla $N^{\circ} 1$, evidenciándose que el $50 \%$ de pacientes presentan el tipo III en donde hay una colangitis esclerosante intrahepática asociada a estenosis papilar. En menor porcentaje se presenta la estenosis papilar, colangitis esclerosante intrahepática única o largas estenosis de las vías biliares extrahepáticas con o sin afección intrahepática ${ }^{5}$.

Volumen 38 | N³ 3 | Diciembre 2020 | Págs. 79-86 


\section{Tabla N¹}

Variantes de la estenosis de la vía biliar asociadas a VIH/SIDA

\begin{tabular}{clc}
\hline Tipo & Patrón en CPRE & \% de casos \\
\hline I & Estenosis papilar & 20 \\
II & Colangitis esclerosante intrahepática única & $15-20$ \\
III & Colangitis esclerosante intrahepática asociada a estenosis papilar \\
IV & $\begin{array}{l}\text { Largas estenosis de las vías biliares extrahepáticas con o sin afección } \\
\text { intrahepática }\end{array}$ & 50 \\
\end{tabular}

Fuente: Tonolini M, Bianco R. ${ }^{7}$

El tratamiento de la colangiopatía asociada al VIH está orientado a controlar los síntomas secundarios a esta patología, el dolor puede ser manejado con derivados opioides en combinación con bloqueo de plexo celíaco. La asociación del ácido ursodexocicólico con la esfinterectomía ha demostrado una mejoría en la función hepática y disminución del dolor abdominal. La terapia antiretroviral cumple un papel crucial en el manejo de la colangiopatía pues se ha demostrado que da un mejor control de las colangitis oportunistas ${ }^{2}$, presentando una reducción de la incidencia de este cuadro clínico hasta un $0.9 \%^{8}$. Si pese al tratamiento aplicado el dolor abdominal y la colestasis aparecen de nuevo es una señal clara de que ya se ha desarrollado una resistencia al tratamiento antirretroviral. Según las revisiones médicas el tratamiento de infecciones oportunistas no causa ningún cambio en el progreso natural de la colangitis esclerosante y la estenosis papilar ${ }^{1,2}$.

El pronóstico de estos pacientes es pobre, pues este cuadro se presenta en estadios avanzados de la inmunosupresión y a su vez las infecciones oportunistas están tomando otros órganos y sistemas. En un año la supervivencia va de 14\% al $41 \%$ y una media de 7 a 12 meses. Dentro de la morbilidad secundaria a esta patología está el desarrollo de un colangiocarcinomas o colangitis esclerosante en donde se da una displasia biliar irreversible y refractaria a la restauración de la inmunidad².
En relación al caso expuesto anteriormente, se trata de un paciente con una colangiopatía asociada a $\mathrm{VIH}$, diagnóstico que fue colocado luego de descartar por medio de estudios de imagen posibles procesos neoplásicos que comprometa las vías biliares u órganos vecinos a la triada portal. El paciente en calidad de portador de este virus por 26 años y junto al tratamiento antirretroviral corresponde al $14 \%$ a $18 \%$ de la población con VIH que van a desarrollar cambios pro inflamatorios del hígado y vías biliares condicionando a una colangiopatía en un período de 3 años ${ }^{1}$, a su vez la literatura describe que el $20 \%$ con VIH suelen presentar signos o síntomas colestásicos aun cuando el recuento de CD4 sea alto mantienen una inmunocompetencia adecuada5; en el presente caso existen valores de CD4 en 728.79 células $/ \mathrm{mm}^{3}$, en donde, pese a que el paciente aun no ha adquirido el síndrome inmunodeficiente, la evidencia demuestra que ya existen alteraciones y patologías de la vía biliar asociada al VIH ${ }^{7}$. Con respecto al tipo de colangiopatía según la clasificación de Tonolini, este caso corresponde al tipo I en donde se evidencia una estenosis papilar a nivel de colangioresonancia y CPRE ${ }^{5}$. Una vez colocado el stent biliar por medio de CPRE se evidenció una evolución favorable llevando a un descenso de su patrón colestásico, resolución de su cuadro clínico y seguimiento médico por consulta externa. 


\section{CONCLUSIONES}

El diagnóstico diferencial de la ictericia obstructiva en el paciente con VIH constituye un reto diagnóstico. La CPRE es diagnóstica y terapéutica ante la ausencia de masa tumoral en los estudios de extensión por imagen. La valoración de casos en comité constituye una herramienta fundamental en la toma de decisiones evitando un procedimiento quirúrgico mayor sin la evidencia imagenológica o histológica de tumor en la triada pancreato biliar en el paciente con $\mathrm{VIH}$.

\section{ABREVIATURAS}

- CD: cúmulo de diferenciación

- Colangiormn: colangioresonancia magnética.

- CPRE: colangiopancreatografía retrógrada endoscópica.

- CA 19.9: antígeno de cáncer 19.9.

- VIH: virus de inmunodeficiencia humana.

- SIDA: síndrome de inmunodeficiencia adquirida.

- md/dl: miligramos por decilitro

- U/L: unidades por litro

- $\mathrm{U} / \mathrm{ml}$ : unidades por mililitro

\section{ASPECTOS BIOÉTICOS}

Este trabajo de investigación fue realizado bajo el previo consentimiento del paciente y con total compromiso de los autores en la confidencialidad del manejo de los datos.

\section{INFORMACIÓN DE LOS AUTORES}

Barzallo Sánchez David Esteban. Médico. Especialista en Cirugía General. Alta especialidad en Cirugía Hepato-PancreatoBiliar. Hospital de Especialidades José Carrasco Arteaga. Unidad de Cirugía General. Cuenca - Azuay - Ecuador. e-mail: davidbarza@gmail.com

ORCID:https://orcid.org/0000-0001-8810-3589

Martínez Gaona Karla Del Cisne. Médica. Universidad de Cuenca. Egresada del Posgrado de Cirugía General. Cuenca - Azuay - Ecuador.

e-mail: karladelcisne@hotmail.com

ORCID:https://orcid.org/0000-0002-4368-3767

\section{CONTRIBUCIÓN DE LOS AUTORES}

Los autores declaran haber contribuido de manera similar en la concepción y diseño del trabajo, análisis e interpretación de los datos, redacción y revisión crítica del manuscrito, aprobación de la versión final y están en capacidad de responder de todos los aspectos del artículo.

\section{CONFLICTO DE INTERESES}

Los autores declaran no tener conflictos de intereses.

\section{FUENTES DE FINANCIAMIENTO}

Autofinanciado.

\section{REFERENCIAS BIBLIOGRÁFICAS}

1. Harshad D, Teena S, Sandeep M. Seetharamu, Dheeraj K. HIV/AIDS cholangiopathy: Clinical spectrum, cholangiographic features and outcome in 30 patients. Journal of Gastroenterology and Hepatology. Internet]. 2010 [citado 26 Ene 2020]; 10 (25): 1656-1660. Disponible en: https://onlinelibrary.wiley.com/doi/ abs/10.1111/j.1440-1746.2010.06336.x

2. Puri P, Kumar S. Liver involvement in human immunodeficiency virus infection. Indian J Gastroenterol. 2016 Jul. 35(4):260-73. doi: 10.1007/s12664-016-0666-8. Epub 2016 Jun 3. PMID: 27256434. https://pubmed. ncbi.nlm.nih.gov/27256434/ 
3. Naseer M, Dailey F, Juboori A, Samiullah $S$, Tahan V. Epidemiology, determinants, and management of AIDS cholangiopathy: A review. World J Gastroenterol. 2018;24(7):767-774. Doi: 10.3748 / wjg. v24.i7.767 https://www.ncbi.nlm.nih.gov/ pmc/articles/PMC5807936/

4. Laguna A, Liza L, Durán E, Díaz J, Jurado J, Pérez J. Diagnóstico por imágenes en el colangiocarcinoma. Sociedad Española de Radiología Médica. Nov. 22, 2018. Presentación Electrónica Educativa. Disponible en: https://piper. espacioseram.com/index.php/seram/ article/download/2026/1011/

5. Mahajani R, Uzer M. Cholangiopathy in HIV infected patients. Clinics in Liver Disease. 1999;3(3):1089-3261 Disponible en: https://www.sciencedirect.com/science/ article/abs/pii/S1089326105700908

6. Romero C, Sanchez C. Protocolo diagnóstico de la colestasis extrahepática. Medicine: Programa de Formación Médica Continuada Acreditado, ISSN 0304-5412, Serie 12. 2016;11: 640-643. Disponible en: https://dialnet.unirioja.es/servlet/ articulo?codigo $=5508451$

7. Tonolini M, Bianco R. HIV-related/ AIDS cholangiopathy: pictorial review with emphasis on MRCP findings and differential diagnosis. Clinical Imaging. 2013; 37: 219-226 Disponible en: https:// www.ncbi.nlm.nih.gov/pubmed/23465971

8. Maweni R Jr, Kallampallil J, Leong S, Akunuri S. Concomitant AIDS cholangiopathy and Fanconi syndrome as complications of HIV in a single patient. BMJ Case Rep. 2017, doi: 10.1136/bcr-2017-222333. https:// pubmed.ncbi.nlm.nih.gov/29167218/
9. Laguna A, Liza L, Durán E, Díaz J, Jurado J, Pérez J. Diagnóstico por imágenes en el colangiocarcinoma. Sociedad Española de Radiología Médica. Nov. 22, 2018. Presentación Electrónica Educativa. Disponible en: https://piper. espacioseram.com/index.php/seram/ article/download/2026/1011/

10. Imai $K$, Misawa $K$, Matsumura $T$, Fujikura Y, Mikita K, Tokoro M, Maeda T, Kawana A. Progressive HIV-associated Cholangiopathy in an HIV Patient Treated with Combination Antiretroviral Therapy. Intern Med. 2016;55(19):2881-2884. doi: 10.2169/internalmedicine.55.6826.1. https://pubmed.ncbi.nIm.nih. gov/27725553/ 